\title{
Entrainment in two coalescing axisymmetric turbulent plumes
}

\author{
Claudia Cenedese ${ }^{1} \dagger$, and P. F. Linden ${ }^{2}$ \\ ${ }^{1}$ Department of Physical Oceanography, Woods Hole Oceanographic Institution, MS\#21, 360 \\ Woods Hole Road, Woods Hole, MA 02543, USA \\ ${ }^{2}$ Department of Applied Mathematics and Theoretical Physics, University of Cambridge, \\ Center for Mathematical Sciences, Wilberforce Road, Cambridge, CB3 0WA, U.K.
}

(Received ?; revised ?; accepted ?. - To be entered by editorial office)

A model of the total volume flux and entrainment occurring in two coalescing axisymmetric turbulent plumes is developed and compared to laboratory experiments. The dynamical evolution of the two plumes is divided into three regions. In Region 1, where the plumes are separate, the entrainment in each plume is unaffected by the other plume, although the two plumes are drawn together due to the entrainment of ambient fluid between them. In Region 2 the two plumes touch each other but are not yet merged. In this region the total entrainment is a function of both the dynamics of the touching plumes and the reduced surface area through which entrainment occurs. In Region 3 the two plumes are merged and the entrainment is equivalent to that in a single plume. We find that the total volume flux after the two plumes touch and before they merge increases linearly with distance from the sources, and can be expressed as a function of the known total volume fluxes at the touching and merging heights. Finally, we define an 'effective' entrainment constant, $\alpha_{e f f}$, as the value of $\alpha$ needed to obtain the same total volume flux in two independent plumes as that occurring in two coalescing plumes. The definition of $\alpha_{e f f}$ allows us to find a single expression for the development of the total volume flux in the three different dynamical regions. This single expression will simplify the representation of coalescing plumes in more complex models, such as in large scale geophysical convection, in which plume dynamics are not resolved. Experiments show that the model provides an accurate measure of the total volume flux in the two coalescing plumes as they evolve through the three regions.

\section{Introduction}

The coalescing of turbulent plumes is a common process in environmental and industrial applications. The air circulation in a ventilated enclosure with multiple heat sources, e.g. offices with electronic equipment and humans, is regulated by the dynamics of turbulent buoyant plumes that rise vertically and interact. The final stratification in these enclosures and the resulting ventilating flow are determined by the entrainment into the interacting plumes (Linden 1999). Turbulent plumes from smokestacks can interact if the stacks are sufficiently close together, and the depth at which the coalescing plumes reach their neutral buoyancy level and intrude horizontally in the stratified atmosphere depends on the details of this interaction. Buoyant turbulent plumes are produced around oceanic ridges from hydrothermal vents (Baker et al. 1995; Speer \& Rona 1989) and they are occasionally observed to coalesce. Coastal cities often discharge their partially treated waste waters at the bottom of the ocean via an outfall. Ocean outfall design is governed

$\dagger$ Email address for correspondence: ccenedese@whoi.edu 
by the dynamics regulating the interaction of multiple buoyant jets (Lai \& Lee 2012; Lee 2012) and their ability to dilute the waste waters. Finally, the dynamics of buoyant vertical plumes has recently been shown to have a important role in the melting of some vertical glacier faces (Jenkins 2011; Xu et al. 2012; Sciascia et al. 2013). At the front of marine terminating glaciers, freshwater plumes are generated by subglacial discharge and submarine melting. The presence of multiple plumes may affect glacial melting ( $\mathrm{Xu}$ et al. 2013; Kimura et al. 2014) through the modified entrainment in the interacting plumes.

Despite these numerous applications, there are relatively few studies focusing on multiple jets and plumes. One of the first studies investigating the interaction between multiple jets focused on the development of clouds (Baines \& Keffer 1974), which was followed by further work focusing on buoyant plumes close to ideal, i.e. with small initial volume and momentum fluxes, by Baines (1983) and Kaye \& Linden (2004), and work focusing on buoyant jets with significant momentum fluxes (Yannopoulos \& Noutsopoulos 2006a,b; Lai \& Lee 2012). In particular, Kaye \& Linden (2004) is the first detailed study on coalescing plumes pair focused on the condition for merging. The current paper extends this work by describing the total volume flux and entrainment evolution in two co-flowing, vertical turbulent coalescing plumes. We restricted attention to Boussinesq plumes so that rising buoyant plumes are dynamically equivalent to descending dense plumes. In particular, we develop a model for the transition region that connects the region in which the two plumes are independent with the region in which the two plumes are merged, and we propose an 'effective' entrainment constant to be used when parameterizing the dynamics of the coalescing plumes. A model for the entrainment into two coalescing plumes is described in $\S 2$. Experiments on the coalescing of two plumes are described and compared with the model in $\S 3$. Our conclusions are given in $\S 4$.

\section{Dynamics of two coalescing plumes}

Consider two axisymmetric vertical turbulent plumes with equal buoyancy fluxes $F=$ $g^{\prime} Q_{p}$, where $g^{\prime}=g\left(\rho_{p}-\rho_{a}\right) / \rho_{0}$ is the reduced gravity, $g$ is the gravitational acceleration, $\rho_{p}$ is the density of the plume, $\rho_{a}$ is the uniform ambient density, $\rho_{0}$ is a reference density, and $Q_{p}$ is the volume flux of each plume, with origins at the same height and source separation $x_{0}$. In a uniform environment, the buoyancy flux in each plume is conserved and $F$ can be calculated at the source where the values of $g^{\prime}$ and $Q_{p}$ are known. Each individual plume is assumed to follow the self-similar solutions proposed by Morton et al. (1956) and the combined behavior of the two plumes can be described in three distinct regions (Figure 1). Near the source each plume will behave independently until they touch (Region 1). In this region, although the two plumes are independent, they do interact by drawing together due to the entrainment of ambient fluid in the region between them. After the two plumes touch, they will interact in a region where the selfsimilar solutions for a single plume do not hold (Region 2). At an even larger distance from the sources the two plumes will have merged into a single plume. As discussed in detail by Kaye \& Linden (2004, henceforth KL04), the merging height is defined to be the height at which the centerline value of the combined average buoyancy profiles of the two turbulent plumes (blue lines in Figure 1), each taken as Gaussian, first becomes a local maximum, i.e. the height at which there are no longer two distinct peaks in the buoyancy profile. In this region, the resulting merged plume can again be described by the self-similar solutions (Region 3). Although the derivation of the merging height and merged plume characteristics have been obtained previously by KL04, they will be briefly summarized below for completeness. The novelty of the present work resides in connecting the characteristics of the two plumes in the previously known Regions 1 and 


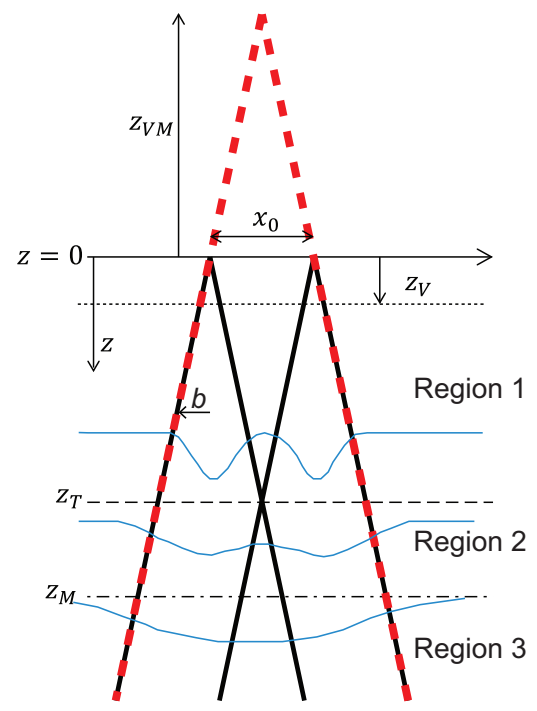

FiguRE 1. Schematic of two descending dense plumes showing the three regions used to describe the dynamics of two coalescing plumes. The red dashed lines indicate the merged plume. The blue lines indicate a schematic of the combined average buoyancy profiles of the two equal turbulent plumes, each taken as Gaussian.

3 through the development of the novel 'transition' Region 2. Furthermore, the present study focuses on the entrainment into the coalescing plumes in the three regions, an aspect that has not been addressed previously.

\subsection{Region 1: Independent plumes}

Consider two axisymmetric turbulent plumes with sources at the same height and separated by a horizontal distance $x_{0}$. When the two plumes are independent of each other the average buoyancy profile of each single turbulent plume can be represented by a 'top hat' profile (Turner 1979) with a radius given by $b(z)=\frac{6}{5} \alpha z$, where $z=\left(z^{\prime}+z_{V}\right), z^{\prime}$ is the vertical distance from the source, $z_{V}$ is the location of the virtual origin (Hunt \& Kaye 2001), and $\alpha$ is the entrainment constant (Morton et al. 1956). The virtual origin correction is the distance from the physical source, that an imaginary pure plume, with zero momentum and volume fluxes but with the same buoyancy flux issuing from the virtual origin, has in order for the actual buoyancy, momentum and volume fluxes of the plume to be the same at the physical source, and is used since the equations introduced below are strictly valid for pure plumes. The volume flux in each plume is given by

$$
Q_{1}=Q_{2}=\left(\frac{9}{10}\right)^{\frac{1}{3}} \frac{6}{5} \pi^{\frac{2}{3}} \alpha^{\frac{4}{3}} F^{\frac{1}{3}} z^{\frac{5}{3}}
$$

and the total volume flux carried by the two plumes is $Q=2 Q_{1}$. If the two plumes did not interact they would touch when the radius of each plume was equal to half the distance between the sources, i.e. $b(z)=x_{0} / 2$, which is satisfied when $z=\frac{5}{12} x_{0} / \alpha=0.42 x_{0} / \alpha$. However, it has been previously observed (KL04) that the two plumes attract each other and it is necessary to consider the entrainment of one plume by the other to derive the rate of change of separation between the centerlines of the two plumes with height. In their derivation, KL04 assumed that the two plumes interact with each other before merging, that the radial growth rate of each plume is unaffected by this process, and that after merging, the two plumes behave as a single axisymmetric plume. Following KL04, before the plumes merge the separation $x(z)$ between the centerlines can be expressed as 


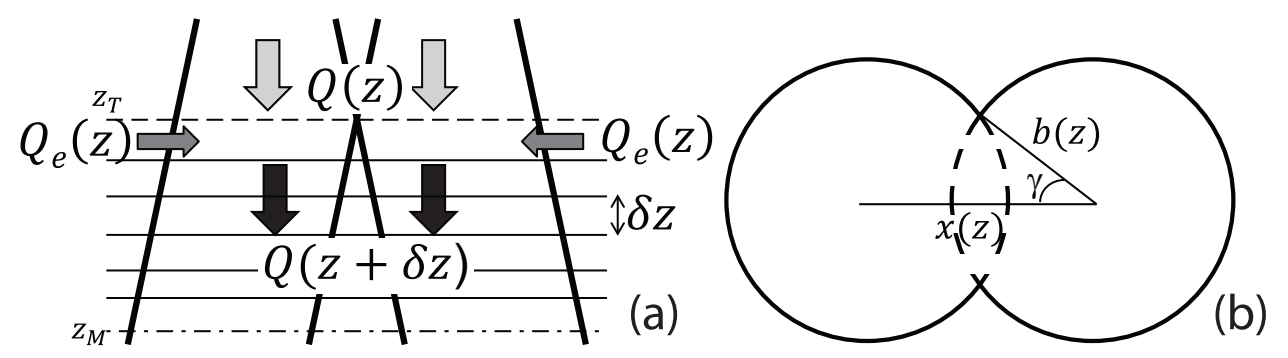

Figure 2. Schematic of the model for Region 2 between $z_{T} \leqslant z \leqslant z_{M}$ where the two plumes do not follow a self-similar solution. ( $a$ ) side view and $(b)$ top view.

(see KL04 for details)

$$
\frac{\partial x}{\partial z}=-\frac{2 \alpha b(z)}{x}
$$

The two plumes will touch when $b(z)=x(z) / 2$, which is satisfied when

$$
z=z_{T}=\sqrt{\frac{25}{204}} \frac{x_{0}}{\alpha}=0.35 \frac{x_{0}}{\alpha}
$$

which is closer to the sources than when the mutual attraction is ignored. When this attraction is included, the distance between the centerlines when the plumes touch is therefore $x=x_{T}=\sqrt{\frac{12}{17}} x_{0}=0.84 x_{0}$. Using $x_{0} / \alpha$ and $x_{0}^{4 / 3} F^{-1 / 3}$ to obtain dimensionless length and time, respectively, the dimensionless volume flux carried by the two plumes becomes

$$
Q^{*}=2\left(\frac{9}{10}\right)^{\frac{1}{3}} \frac{6}{5} \pi^{\frac{2}{3}} \alpha^{\frac{8}{3}} z^{* \frac{5}{3}}
$$

where ${ }^{*}$ indicates dimensionless variables, and $z_{T}^{*}=0.35$.

\subsection{Region 3: Merged plume}

Two equal plumes with origins at the same height were theoretically predicted and observed to merge at a distance $z_{M}=\sqrt{\frac{25}{132}} x_{0} / \alpha=0.44 x_{0} / \alpha$ (KL04) from the virtual origin of each individual plume. Further, the resulting merged plume was also predicted and observed experimentally to follow the self-similar behavior of a single plume having twice the buoyancy flux of the individual plumes and a virtual origin which is located at a distance $z_{V M}=0.12 x_{0} / \alpha$ from the virtual origin of the individual plumes (Figure 1 , red dashed line). The distance between the two centerlines when the plumes merge is therefore $x=x_{M}=\sqrt{\frac{6}{11}} x_{0}=0.74 x_{0}$, and the total volume flux carried by the two merged plumes is

$$
Q=\left(\frac{9}{10}\right)^{\frac{1}{3}} \frac{6}{5} \pi^{\frac{2}{3}} \alpha^{\frac{4}{3}}(2 F)^{\frac{1}{3}}\left(z+z_{V M}\right)^{\frac{5}{3}} .
$$

Using the same dimensionless variable as in $\S 2.1$, the dimensionless volume flux carried by the two merged plumes becomes

$$
Q^{*}=2^{\frac{1}{3}}\left(\frac{9}{5}\right)^{\frac{1}{3}} \frac{6}{5} \pi^{\frac{2}{3}} \alpha^{\frac{8}{3}}\left(z^{*}+0.12\right)^{\frac{5}{3}},
$$

and $z_{M}^{*}=0.44$.

\subsection{Region 2: Touching plumes}

In this section a new model is developed to determine, in Region 2, the rate of change of the volume flux with height of the two interacting plumes after they touch and before 

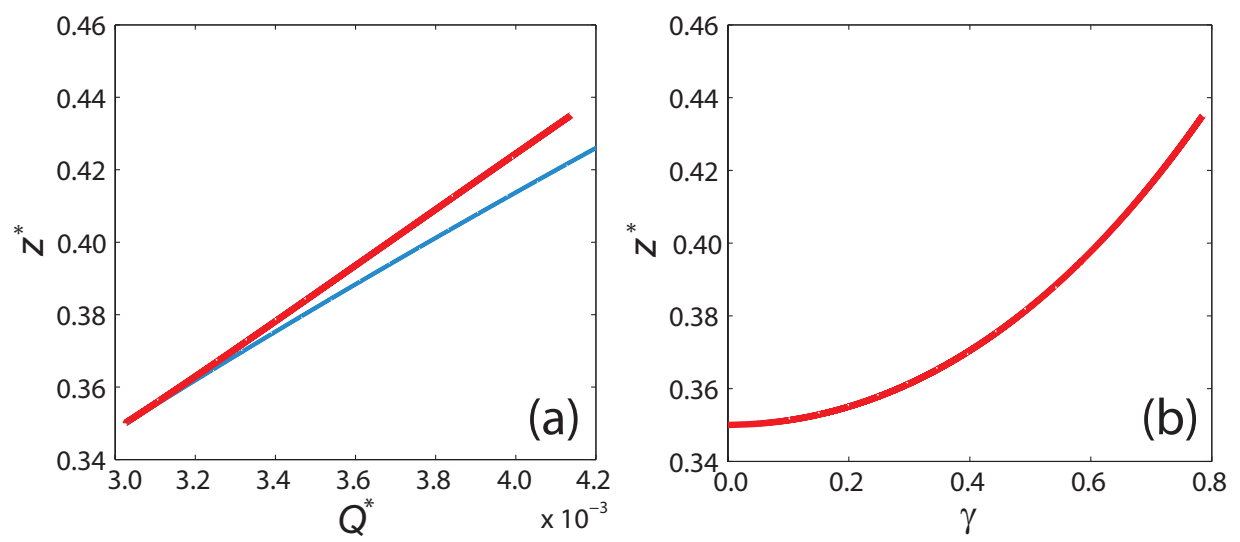

Figure 3. Region 2 (a) dimensionless volume flux for two coalescing (red) and independent (blue) plumes and $(b)$ angle $\gamma$ (in radians) as a function of dimensionless depth.

they merge, i.e. for $z_{T} \leqslant z \leqslant z_{M}$ (Figure 1 ). We assume that in Region 2 the two plumes interact with each other before merging, that the radial growth rate of each plume is unaffected by this process, and that after merging, the two plumes behave as a single axisymmetric plume. This assumption is correct asymptotically but near the interface between Regions 2 and 3 the shape of the interacting plumes may be more similar to that depicted in Figure 2b. Considering a horizontal slice through the two interacting plumes (Figure 2b), the rate of change of the volume flux can be calculated by assuming conservation of mass and by assuming that the radial expansion of each plume follows the isolated plume value $b(z)=\frac{6}{5} \alpha z$. The volume flux exiting the bottom of the horizontal slice, $Q(z+\delta z)$, is given by the volume flux entering at the top of the horizontal slice, $Q(z)$, plus the volume flux entrained through the boundaries of the plumes, $Q_{e}(z)$ (Figure $2 \mathrm{a})$. With the assumption that the mean entrainment velocity across the edge of the plumes is proportional to the local mean downward velocity (Morton et al. 1956), i.e. $w_{e}(z)=\alpha w(z)$, where $w(z)=Q(z) / A_{p}(z)$ and $A_{p}$ is the horizontal area covered by the two interacting plumes, the entrainment flux is given by $Q_{e}(z)=P_{p}(z) w_{e}(z) \delta z$, where $P_{p}$ is the perimeter of the edges of the two interacting plumes (thick solid line in Figure $2 \mathrm{~b}$ ) and $\delta z$ is the height of the horizontal slice. Hence, the rate of change of the volume flux carried by the two plumes between $z_{T} \leqslant z \leqslant z_{M}$ is given by

$$
\frac{\partial Q}{\partial z}=\alpha Q(z) \frac{P_{p}(z)}{A_{p}(z)}
$$

The expressions for $P_{p}(z)$ and $A_{p}(z)$ can be found by defining $\gamma$ as the angle between the line connecting the centers of the plumes and the radius $b(z)$ of the plume passing through the overlap point between the two plumes (see Figure $2 \mathrm{~b}$ )

$$
\begin{gathered}
P_{p}(z)=4 b(z)(\pi-\gamma(z)), \\
A_{p}(z)=b(z)^{2}(\sin (2 \gamma(z))+2(\pi-\gamma(z))) .
\end{gathered}
$$

It is worth noting that equation (2.7) is general and can be applied also to a single axisymmetric plume to derive equation (2.1). However, hereafter we will be using (2.7) to derive the volume flux of two coalescing plumes in Region 2. The numerical solution of equations (2.7)-(2.9) suggests that the volume flux increases linearly with $z$ in Region 2 as $\gamma$ increases from $0^{\circ}$ to $45^{\circ}$ (Figure 3). Hence, given the known volume fluxes at $z_{T}$ 

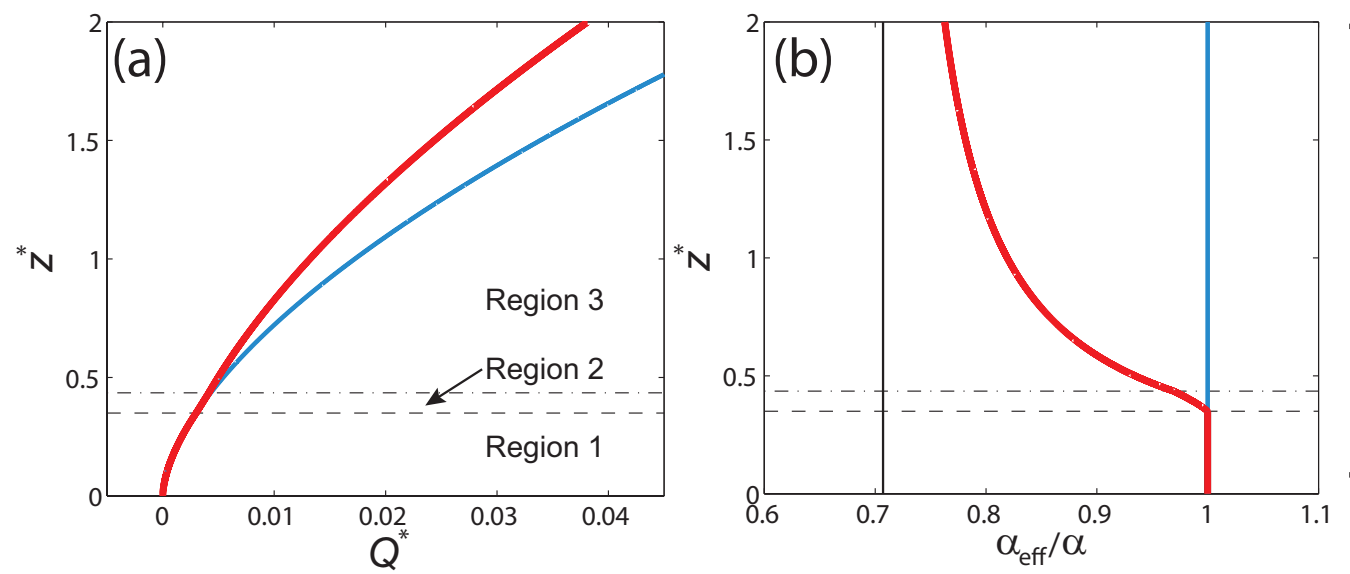

FiguRE 4. Dependence of (a) the total volume flux for two coalescing (red) and independent (blue) plumes and $(b)$ the effective entrainment parameter with depth. Dashed and dot-dashed lines represent the touching height, $z_{T}$, and the merging height, $z_{M}$, respectively. Thin black line indicates the value of $\alpha_{e f f} / \alpha$ for $z^{*} \rightarrow \infty$.

and $z_{M}$, namely $Q_{T}$ and $Q_{M}$, the volume flux in Region 2 can be expressed as

$$
Q=\frac{Q_{M}-Q_{T}}{z_{M}-z_{T}} z+\frac{Q_{T} z_{M}-Q_{M} z_{T}}{z_{M}-z_{T}} .
$$

The approximation introduced using equation (2.10) in place of the numerical solution of equations (2.7)-(2.9) is small, as indicated by the least square fit of the two solutions having a slope of 1.004 and an $R^{2}=0.99$. Note that the linearity of the solution of equations (2.7)-(2.9), which is a result of the geometric dependence of $\gamma$ on $z$, does not hold for a single axisymmetric plume (Figure 4a).

\section{4. 'Effective' entrainment}

The total volume flux in the coalescing plumes increases with depth but the rate of change decreases as the two plumes move from Region 1 to Region 3 (Figure 4a). Since the variation of total volume flux with depth is known, we can then define an 'effective' entrainment constant, $\alpha_{e f f}$, as the value of $\alpha$ needed to obtain the same total volume flux in two independent plumes as that occurring in two merging plumes, i.e. by comparison with $(2.1)$

$$
Q=2\left(\frac{9}{10}\right)^{\frac{1}{3}} \frac{6}{5} \pi^{\frac{2}{3}} \alpha_{e f f}^{\frac{4}{3}} F^{\frac{1}{3}} z^{\frac{5}{3}} .
$$

Using the expression of $Q$ in each region we find

$$
\frac{\alpha_{e f f}}{\alpha}= \begin{cases}1, & z^{*}<0.35 \\ z^{*-\frac{5}{4}}\left(0.73 z^{*}-0.082\right)^{\frac{3}{4}}, & 0.35 \leqslant z^{*} \leqslant 0.44 \\ \frac{1}{2^{\frac{1}{2}}}\left(1+\frac{0.12}{z^{*}}\right)^{\frac{5}{4}}, & z^{*}>0.44,\end{cases}
$$

In equation (2.11) the merged plume virtual origin is not present explicitly since it is included in the expression of $\alpha_{e f f}$ in Region 3. The value of $\alpha_{e f f}$ in Region 1 is by definition equal to $\alpha$, and is also the maximum value of the entrainment coefficient, since two independent plumes entrain more than two merged plumes. After reaching the location where the two plumes touch, the effective entrainment then decreases monotonically in Regions 2 and 3. The entrainment in the merged plume (red line in Figure 4b), 


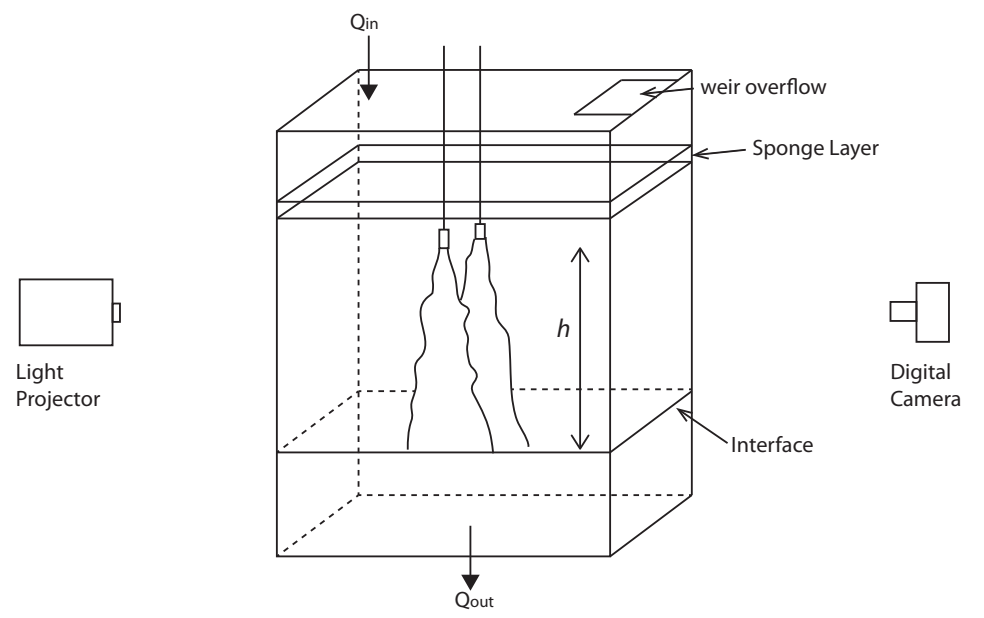

Figure 5. Schematic showing the experimental set up. Not to scale. The light projector was located at $650 \mathrm{~cm}$ from the tank and the digital camera at $263 \mathrm{~cm}$ from the tank.

compared to the entrainment in the two independent plumes (blue line in Figure 4b), decreases with increasing distance from the merging height. Hence, the two plumes are expected to entrain more ambient fluid the farther apart they are.

\section{Experiments}

Experiments were performed to test the validity of the model presented in $\S 2$, in particular the novel derivation of the plumes behavior in Region 2 and the expression for $\alpha_{\text {eff }}$. The experimental set up was similar to KL04. Two salt solution plumes were located in a tank $\left(40 \times 40 \times 70 \mathrm{~cm}^{3}\right)$ filled with fresh water (Figure 5). In order to generate turbulent plumes from the sources, the same special nozzles (designed by Dr. Paul Cooper) used by KL04 were employed. The flow rate and density of the salt solution were chosen such that the turbulent plumes had a small initial volume and momentum flux, which made them close to ideal. The virtual origin for the individual plumes, $z_{V}$, and the entrainment constant $\alpha$ associated with the plume sources were determined by using a slight modification of the 'filling box' technique (see $\S 3.1$ ). Two flow rates were used, $Q_{0}=1.38 \mathrm{~cm}^{3} \mathrm{~s}^{-1}$ and $Q_{0}=2.18 \mathrm{~cm}^{3} \mathrm{~s}^{-1}$, where $Q_{0}$ is the flow rate of each individual plume, and the reduced gravity was $g_{0}^{\prime}=9.8 \mathrm{~cm} \mathrm{~s}^{-2}$ and $g_{0}^{\prime}=13.5 \mathrm{~cm} \mathrm{~s}^{-2}$, respectively, to obtain two different buoyancy fluxes $F=13.5 \mathrm{~cm}^{4} \mathrm{~s}^{-3}$ and $F=29.4 \mathrm{~cm}^{4} \mathrm{~s}^{-3}$. The separation of the two sources took the values $x_{0}=2.0,3.0,4.0,5.0,6.0,7.0,7.8 \mathrm{~cm}$.

\subsection{Experimental technique}

In order to measure the total flow rate within the two plumes and, consequently, the rate of change of the entrainment occurring in the three different regions, the 'filling box' technique described in Baines (1983) was employed. The tank was supplied with fresh water at the surface through a foam float (in order to minimize disturbances near the free surface) and a long weir was positioned near the free surface to keep it at the same location throughout the experiment. A pump withdrew water at the bottom of the tank. The two plume sources were located in the upper part of the tank (Figure 5 ), and a sponge layer was positioned above the sources to minimize any motion due to the continuous supply of fresh water at the free surface. The salt solution was dyed 
with food coloring. After starting the flow of salt water through the plume sources, part of the plume water exited the tank and part started accumulating near the bottom of the tank. The flow rate of the exiting water was regulated to obtain a sharp interface approximately $20 \mathrm{~cm}$ from the sources, as determined by a shadowgraph image. To create this image a light projector was positioned $650 \mathrm{~cm}$ from the tank and a video camera was located $262 \mathrm{~cm}$ from the opposite side of the tank. In the classic 'filling box' technique the height of the stationary interface is recorded for different exiting flow rates which are then equivalent to the total flow rate in the plumes at the interface (see Baines (1983) for details). In the present experiments, we also took a digital video of the experiment after the pump withdrawing the water at the bottom of the tank was shut off. At the same time the supply of fresh water from the free surface was also halted. The interface between the plume waters (dyed red) and the ambient fresh water rose in time and the total flow rate in the two plumes at the interface depth is given by

$$
Q(z=h)=\left(A-A_{p}(z)\right) \frac{\partial h}{\partial t},
$$

where $h$ is the distance of the interface from the virtual origin of the individual plumes, and $A$ is the cross sectional area of the tank. For simplicity, for the remainder of the paper we will assume $A_{p}(z)=0$. Since $A_{p} \leqslant 0.05 A$ in the present configuration, this approximation has a negligible effect on the results. Time-series of the interface positions were constructed from video of the experiments by averaging vertical slices, from each frame, which were not occupied by the plumes. The position of the interface was then identified by a change in the brightness between the dyed plume waters and the ambient fluid, and the threshold used was between $85 \%$ and $75 \%$ of the brightness difference between the two fluids.

\subsection{Experimental results}

Experiments were conducted with different buoyancy fluxes and source separations. The position of the interface distance from the virtual origin in time, $h(t)$, was obtained for each experiment as illustrated in Figure 6a. To compare the laboratory results to the theoretical prediction (Figure 6b), we substitute (2.1), (2.5) and (2.10) into (3.1) and consider the following non-dimensional variables

$$
\lambda=\frac{\alpha h}{x_{0}}, \tau=\frac{t-t_{T}}{A x_{0}^{-\frac{2}{3}} F^{-\frac{1}{3}}},
$$

where $t_{T}$ is the time at which the interface is at the touching height, i.e. $h\left(t_{T}\right)=z_{T}$, to obtain the evolution equation for the dimensionless interface height

$$
\lambda(\tau)= \begin{cases}{\left[0.35^{-\frac{2}{3}}+\frac{4}{3}\left(\frac{9}{10}\right)^{\frac{1}{3}} \frac{6}{5} \pi^{\frac{2}{3}} \alpha^{\frac{2}{3}} \tau\right]^{-\frac{3}{2}},} & \lambda<0.35 \\ \frac{1}{a^{\prime}}\left[\left(0.35 a^{\prime}+b^{\prime}\right) e^{-\frac{a^{\prime}}{A} \tau}-b^{\prime}\right], & 0.35 \leqslant \lambda \leqslant 0.44 \\ {\left[(0.44+0.12)^{-\frac{2}{3}}+\frac{2^{\frac{4}{3}}}{3}\left(\frac{9}{10}\right)^{\frac{1}{3}} \frac{6}{5} \pi^{\frac{2}{3}} \alpha^{\frac{2}{3}}\left(\tau-\delta t^{*}\right)\right]^{-\frac{3}{2}}-0.12,} & \lambda>0.44,\end{cases}
$$

where $\delta t^{*}$ is the dimensionless time it takes the interface to move from $z=z_{T}$ to $z=z_{M}$

$$
\delta t^{*}=\int_{z_{T}}^{z_{M}} \frac{1}{t^{\prime}} \frac{A}{Q(z)} \mathrm{d} z=\frac{A}{a^{\prime}} \ln \frac{Q_{M}}{Q_{T}}=0.34
$$

and is observed in the experiments to be approximately constant and equal to 0.35 , in excellent agreement with the theoretical prediction (3.4). Here $a^{\prime}=a t^{\prime}$ and $b^{\prime}=b t^{\prime} / h^{\prime}$, where $h^{\prime}=x_{0} / \alpha$ and $t^{\prime}=A x_{0}^{-\frac{2}{3}} F^{-\frac{1}{3}}$ are the length and time scales, respectively, used to 

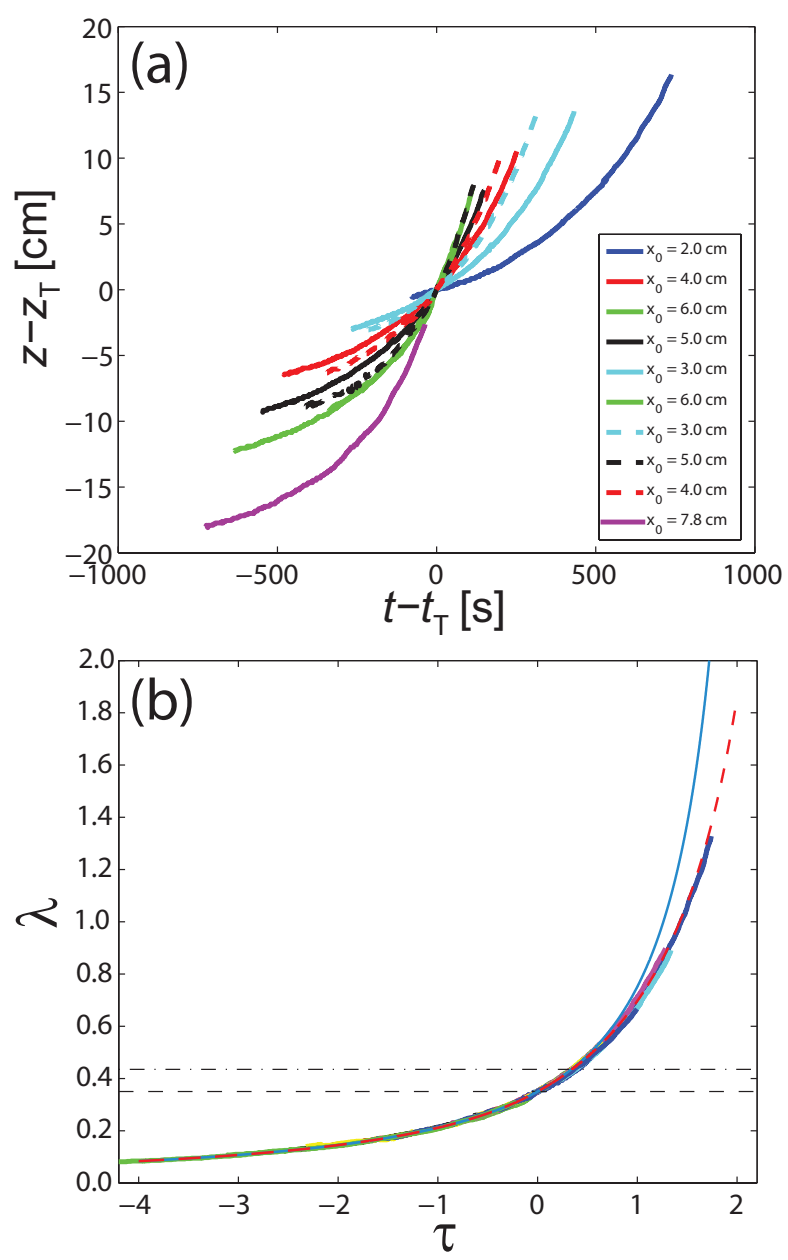

FiguRE 6. (a) Distance of the interface $h$ from the virtual origin, as a function of time for the laboratory experiments; solid and dashed colored lines indicate experiments with $F=13.5 \mathrm{~cm}^{4}$ $\mathrm{s}^{-3}$ and $F=29.4 \mathrm{~cm}^{4} \mathrm{~s}^{-3}$, respectively. (b) Dimensionless values (colored lines) and theoretical prediction (3.3) (thin dashed red line) of $h$; the thin blue line indicates the theoretical prediction of $h$ for two independent plumes; black dashed and dot-dashed lines indicate the dimensionless position of $z_{T}$ and $z_{M}$, respectively.

obtain the dimensionless variables (3.2), and $a$ and $b$ are the slope and intercept of the straight line (2.10). As shown in Figure 6b, this re-scaling provides a complete collapse of the data onto a single curve and (3.3) is an excellent fit to the experiments conducted with different buoyancy fluxes and distances between the sources. With the exception of the experiment with $x_{0}=7.8 \mathrm{~cm}$, the other nine experiments extend through the entire depth of Region 2 with a maximum value of $\lambda$ ranging from 0.44 to 1.33, i.e. $\lambda \geqslant z_{M}^{*}=0.44$. Hence, $(2.11)$ and $(2.12)$ can be used to determine the total volume flux and entrainment in two coalescing plumes and their evolution with depth. The advantage of using these expressions is that, given the buoyancy flux of the two plumes and their separation distance, the development of the volume flux in the three different dynamical regions can be represented by a single equation, making it easier to implement in numerical models or parameterizations of coalescing plumes. 


\section{Conclusions}

We have considered theoretically and experimentally the volume flux and entrainment occurring in two coalescing axisymmetric turbulent plumes. A model was developed to calculate the rate of change of the volume flux after the two plumes have touched and before they have merged. This region was investigated for multiple turbulent buoyant jets using a superposition approach by Yannopoulos \& Noutsopoulos $(2006 a, b)$ and Lai \& Lee (2012), but its effects appear to have been ignored since it was observed to be smaller in vertical extent than the region over which the two plumes draw together (Baines 1983, KL04). The present model predicts that the total volume flux in the two touching plumes increases linearly between $z_{T} \leqslant z \leqslant z_{M}$. We derive an expression for the volume flux (2.10) in this region consistent with the known volume fluxes at the two boundaries, $z=z_{T}$ and $z=z_{M}$. The extension of this transition region where the two plumes are touching but not yet merged, is $0.09 x_{0} / \alpha$, approximately a quarter of the extent of Region $1\left(z_{T}=0.35 x_{0} / \alpha\right)$. This theoretical estimate is in agreement with both our laboratory experiments and previous laboratory experiments of KL04, who observed the transition region to cover a vertical distance of the order of the source separation. Hence, Region 2 is undoubtedly smaller than Region 1, but a rigorous description of the characteristics of the two plumes in this region is necessary for a correct representation of the total entrainment.

The model of the vertical evolution of the total volume flux in two coalescing plumes in the three dynamically relevant regions was tested experimentally. The experimental results are consistent with the location of the touching height, $z_{T}$, and the distance between $z_{T}$ and $z_{M}$ which defines the novel Region 2, as shown by the collapse of the experimental data in Figure $6 \mathrm{~b}$.

Once the evolution of the total volume flux as a function of depth is known, we define an 'effective' entrainment constant, $\alpha_{e f f}$, as the value of $\alpha$ needed to obtain the same total volume flux in two independent plumes as that occurring in two merging plumes (2.11) and an expression for $\alpha_{e f f}$ is found (2.12). The definition of a single equation for the evolution of the volume flux in two coalescing plumes (2.11) simplifies the representation of the dynamics in more complex models in which the plumes are not resolved.

The model presented is valid only when the two plumes have the same buoyancy flux and the same source height. In numerous applications these limitations may not apply and further work is necessary to extend the current model to a more general setting.

The authors gratefully acknowledge the National Science Foundation (Grant OCE0824636) and the Office of Naval Research (Grant N00014-09-1-0844) for their support of the 2013 WHOI Geophysical Fluid Dynamics Summer School where this project was initiated. Support to CC was given by the National Science Foundation project OCE1130008. CC wishes to thank Jason Hyatt for improving the clarity of this manuscript.

\section{REFERENCES}

Baines, W. D. 1983 A technique for the direct measurement of volume flux of a plume. J. Fluid Mech. 132, 247-256.

Baines, W. D. \& Keffer, K. F. 1974 Entrainment by a multiple source turbulent jet. Adv. Geophys. 18(B), 289-298.

Baker, E. T., German, C. R. \& Elderfield, H. 1995 Hydrothermal plumes over spreadingcenter axes: Global distributions and geological inferences. In Seafloor Hydrothermal Systems: Physical, Chemical, Biological, and Geological Interactions (ed. S. E. Humphris, R. A. Zierenberg, L. S. Mullineaux \& R. E. Thomson), Geophys. Monogr. Ser., vol. 91, pp. 47-71. American Geophysical Union. 
Hunt, G. R. \& Kaye, N. B. 2001 Virtual origin correction for lazy turbulent plumes. J. Fluid Mech. 435, 377-396.

JEnkins, A. 2011 Convection-driven melting near the grounding lines of ice shelves and tidewater glaciers. Journal of Physical Oceanography 41, 2279-2294.

Kaye, N. B. \& Linden, P. F. 2004 Coalescing axisymmetric turbulent plumes. J. Fluid Mech. 502, 41-63.

Kimura, S., Holland, P.R., Jenkins, A. \& Piggott, M 2014 The effect of meltwater plumes on the melting of a vertical glacier face. Journal of Physical Oceanography Submitted, $0-0$.

LAi, A. C. H. \& LeE, J. H. W. 2012 Dynamic interaction of multiple buoyant jets. J. Fluid Mech. 708, 539-575.

LEE, J. H. W. 2012 Mixing of multiple buoyant jets. Journal of Hydraulic Engineering 138, 1008-1021.

Linden, P. F. 1999 The fluid mechanics of natural ventilation. Annu. Rev. Fluid Mech. 31, 201-238.

Morton, B. R., Taylor, G. I. \& Turner, J. S. 1956 Turbulent gravitational convection from maintained and instantaneous sources. Proc. R. Soc. Lond. 234, 1-23.

Sciascia, R., Straneo, F., Cenedese, C. \& Heimbach, P. 2013 Seasonal variability of submarine melt rate and circulation in an east greenland fjord. Journal of Geophysical Research: Oceans 118 (5), 2492-2506.

Speer, K. G. \& Rona, P. A. 1989 A model of an atlantic and pacific hydrothermal plume. Journal of Geophysical Research: Oceans 94 (C5), 6213-6220.

Turner, J. S. 1979 Buoyancy Effects in Fluids. New York: Cambridge University Press.

Xu, Y., Rignot, E., Fenty, I., Menemenlis, D. \& Flexas, M. M. 2013 Subaqueous melting of store glacier, west greenland from three-dimensional, high-resolution numerical modeling and ocean observations. Geophysical Research Letters 40 (17), 4648-4653.

Xu, Y., Rignot, E., Menemenlis, D. \& Koppes, M.N. 2012 Numerical experiments on subaqueous melting of Greenland tidewater glaciers in response to ocean warming and enhanced subglacial runoff. Annals of Glaciology 53 (60), 229-234.

Yannopoulos, P. C. \& Noutsopoulos, G. C. $2006 a$ Interaction of vertical round turbulent buoyant jets - Part 1: Entrainment restriction approach. Journal of Hydraulic Research $44(2), 218-232$.

Yannopoulos, P. C. \& Noutsopoulos, G. C. $2006 b$ Interaction of vertical round turbulent buoyant jets - Part 2: Superposition method. Journal of Hydraulic Research 44 (2), 233248 . 